\title{
A New Nano-Platform for Drug Release via Nanotubular Aluminum Oxide
}

\author{
Kunbae Noh ${ }^{1}$, Karla S. Brammer ${ }^{1}$, Chulmin Choi ${ }^{1}$, Seung Hyun Kim ${ }^{2}$, Christine J. Frandsen ${ }^{1}$, \\ Sungho Jin ${ }^{1}$
}

${ }^{1}$ Materials Science \& Engineering, University of California, San Diego, USA; ${ }^{2}$ Bioengineering Department, University of California, San Diego, USA.

Email: jin@ucsd.edu

Received April 12 ${ }^{\text {th }}, 2011$; revised May 10 2011 ; accepted May $25^{\text {th }}, 2011$.

\begin{abstract}
Nanotubular materials have many favorable properties for drug delivery. We present here a pioneering study of controlled release of a model drug, amoxicillin, from the internal nanopore structure of self-ordered, periodically spacedapart aluminum oxide with an innovative, nanotubular geometry. This aluminum oxide nanotube geometry has not yet been revealed for biological applications, thus we have selected this oxide nanotube structure and demonstrated its ability as a drug carrier. Controlled, sustained release was achieved for over 5 weeks. The release kinetics from the nanotube layer was thoroughly characterized and it was determined that the amount of drug released was proportional to the square root of time. This type of controlled release and longevity from the nanotube layer has potential for therapeutic surface coatings on medical implants. Furthermore, this type of geometry has many features that are advantageous and biologically relevant for enhancing tissue biointegration.
\end{abstract}

Keywords: AAO, Nanotube, Antibiotics, Drug Release, Implant Surface

\section{Introduction}

Recently, anodic aluminum oxide (AAO) has become one of the most popular self-ordered periodic, porous templates. In general the highly developed, superior ordering of nanopores in AAO templates is obtained by using a two-step anodization process [1], a rather simple processing method. The AAO porous structure can be uniquely altered based on processing parameters and both porous and tubular shapes can be achieved and tailored with pore diameters between $5 \mathrm{~nm}-10 \mu \mathrm{m}$ and film thicknesses (vertical height) reaching over $100 \mu \mathrm{m}$ [2]. AAO has been of great interest due to its outstanding material properties, including electrical insulation, optical transparency and chemical stability, and most recently because of its biologically inert and biologically compatibility properties [2]. In terms of biological applications, the characteristic periodic porous films of AAO has been used for encapsulating enzymes [3], implant surface coatings on Ti alloys for bone in growth [4-5], membranes for hemodialysis [6], cardiovascular stent applications [7,8], biofiltration [9], and drug delivery $[10,11]$.

Owing to porous AAO's ability to mimic the dimen- sions and nanoporous structural components of natural bone and the prospect of housing genes or drugs for therapeutic treatments within the pores [11], AAO films can be seen as promising coatings for medical, particularly orthopedic, implants. Research on biomedical applications of both porous alumina, and along the same lines nanotubular titania $\left(\mathrm{TiO}_{2}\right)$ [12-18], has increased tremendously in the past few years. It is well known that titania nanotube surfaces elicit favorable properties for bone cell growth and mineralization in vitro $[16,18]$, osseointegration (bone/implant interface bonding) in vivo [19], enhance differentiation of mesenchymal stem cells toward osteogenic maturation [17], as well as elicit long term drug release [15]. Titania nanotubes have been recently recognized for their impact in the future of nanomedicine [20].

We focused on hard-anodized AAO which is recently of great interest because of its advantageous characteristics, i.e. 1) AAO film grows rapidly 1) it is well ordered and 2 ) there is a wide range of novel AAO nanostructures that can be obtained during the hard anodized AAO region which are yet to be discovered [21-24]. For example, Zhao et al. reported a special AAO structure grown 
under constant current anodization, which is represented by a six-membered ring symmetry around the center pores with a regular hexagonal pore arrangement.

While nanopore configuration has been studied greatly for potential biomedical and drug delivery type applications, the nanotube configuration has a unique feature in that the $10-20 \mathrm{~nm}$ spacing between neighboring nanotubes allow a continuous supply of body fluids, nutrients and proteins underneath adhered and growing cells that could potentially aide in tissue development and biointegration. In contrast, simple nanopored configuration gets covered with adhered cells thus blocking the supply of body fluid. This has been described for the case of $\mathrm{TiO}_{2}$ nanotubes with significantly enhanced osteoblast (bone cell) and bone tissue growth ${ }^{16}$, and applies to other adhered cells on the nanotubes.

To imitate the $\mathrm{TiO}_{2}$ nanotube geometry, we have microstructurally altered traditional porous AAO to give rise to a unique nanotube morphology to determine its use in biological applications. It is projected that we can utilize the nanotubes as "nanodepots" for advanced drug delivery therapeutics. AAO nanotubes are an example of a multidisciplinary approach for combining nanotechnology, biomedical engineering, and controlled drug delivery where antibiotics, growth factors, etc. are appropriately needed as well as proper biointegration (osseointegration for example) is desired [11]. The nanotube geometry, as compared to the nanopore geometry with comparable pore diameter, provides $\sim 2 \mathrm{X}$ more surface area, which could be utilized to provide more active spots and functional conjugation locations for increased adsorption or biomolecules, proteins, enzymes, and drug molecules.

The use of AAO nanotubes is a new clinical approach not only for orthopedics, but also for the treatment of several other drug eluting implants which ideally would release for longer periods of time, on the order of days, weeks, even months. The aim of this study is to elucidate the complex phenomenon of drug release from AAO nanotube drug carriers. Detailed, characterized release curves, accumulation plots, and kinetics were revealed for the antibiotic drug, amoxicillin. This type of geometrical structure of AAO is novel in itself, but also the time scale of prolonged release of amoxicillin from the AAO nanostructure is incredibly long, on the order of weeks, with a possibility of extending the drug release to much longer periods.

\section{Materials and Methods}

\subsection{Aluminum Pretreatment}

$0.5 \mathrm{~mm}$ thick Al foil purchased from Alfar aesar (99.99\%) was used as a starting material. The Al foil was succes- sively degreased with isopropyl alcohol and acetone for 5 minutes with ultrasonication followed by a D.I. water rinse and a nitrogen gas blow. Then, the organic-free $\mathrm{Al}$ foil was slightly etched in $1 \mathrm{M} \mathrm{NaOH}$ aqueous solution to remove any surface contamination before the electropolishing process. Conducting $\mathrm{Cu}$ tape was used as the electrode pathway to the Al foil and selected portions of the Al foil, such as the edges or backsides, were protected by a lacquer (Miccroshield) in order to make it resistant to the electrolyte used. A mirror-shiny Al foil was obtained after electropolishing in a mixed solution of $\mathrm{HClO}_{4}$ and Ethanol $(1: 4(\mathrm{v} / \mathrm{v}))$ at $5^{\circ} \mathrm{C}$ under $20 \mathrm{~V}$ for ten minutes with a Pt counter electrode. For a control sample, electropolished Al was used in the experiments. For the experimental nanotube samples anodization was conducted.

\subsection{Anodization}

Prior to hard anodization, mild-anodized porous AAO ( $\sim 800 \mathrm{~nm}$ thick layer) was formed under $25 \mathrm{~V}$ in $0.3 \mathrm{M}$ sulfuric acid and then the electrolyte concentration was changed from $0.3 \mathrm{M}$ to $0.06 \mathrm{M}$. Next, anodization voltage was increased (at a rate of $\sim 0.5 \mathrm{~V} / \mathrm{s}$ ) from $25 \mathrm{~V}$ to $35 \mathrm{~V}$ in order to inhibit local AAO film thickening due to localized high current concentration which otherwise would lead to inhomogeneous oxide film growth or even dielectric breakdown during hard anodization. Power supply (Agilent; E3612A) was connected to digital multimeter (Keithley; 2100) to monitor voltage-current evolution during anodization.

\subsection{AAO Post-Treatment}

After the anodization process, the top side of AAO layer was attached to Si substrate with adhesive glue for handling purpose. Then, Al substrate was selectively removed with a mixed $\mathrm{HCl}$ and $\mathrm{CuCl}_{2}$ solution for ten minutes when the reaction ends. Any residual $\mathrm{Cu}$ debris adhered to the bottom of the AAO barrier layer was removed by placing in nitric acid for a few seconds and washed in D.I. water immediately after. The AAO barrier layer was then removed by $5 \mathrm{wt} \%$ phosphoric acid for ten minutes to two hours depending upon barrier layer thickness of the as-grown AAO and observed under Scanning Electron Microscope (SEM; Phillips XL30 ESEM). All samples were cut into identical size pieces $\left(1 \times 1 \mathrm{~cm}^{2}\right)$ and autoclaved before use as drug carriers.

\subsection{Porosity and Surface Area Calculation}

In order to characterize the AAO nanotube film structure, a porosity and surface area calculation was carried out. A theoretical porosity can be computed geometrically based on structural parameters such as pore size and inter pore distance assuming ideal hexagonal arrangement. The fo- 
llowing equation, Equation (1), was used to approximate the porosity, $P$, of the samples,

$$
P=\frac{2 \pi}{\sqrt{3}}\left(\frac{r}{D_{\mathrm{int}}}\right)^{2}
$$

where $r$ is the inner pore radius and $D_{\text {int }}$ is the inter pore distance. The overall surface is assumed to be filled with $\mathrm{Al}_{2} \mathrm{O}_{3}$ except the empty pore space, which is somewhat complex in the case of a tubular structure. The apparent porosity can be calculated based on pore area of the unit cell and divide by unit cell area. To determine the surface area of the samples with an area of $1 \mathrm{~cm}^{2}$, first the nanotube density was established based on SEM images by counting the number of nanotubes per field for a given area. The nanotube density, $N$, was $\sim 2.19 \times 10^{10}$ (nanotubes $/ \mathrm{cm}^{2}$ ). The following equation, Equation (2), was used to approximate the surface area, $S A$, of the samples

$$
S A=\left(2 \pi\left(R^{2}-r^{2}\right)+2 \pi h(R+r)\right) * N
$$

where $R$ is the outer tube radius, $r$ is the inner pore radius, and $h$ is the height or length of the film. This equation is based on the surface area of a tube multiplied by the number of tubes in the sample area. This is a theoretical estimation. It may be that the voids located around the center pore are not completely void the length of the tube, nonetheless $S A$ is dramatically increased based on the introduction of tubes on the surface.

For a $1 \mathrm{~cm}^{2}$ sample, the porosity and surface area was calculated to be $25 \%$ and $1830 \mathrm{~cm}^{2}$, respectively.

\subsection{Antibiotic Loading, Release, and Collection}

Insertion of liquid into AAO nanotubes is not always easy as the surface tension of the liquid has to be overcome. At room temperature $\left(25^{\circ} \mathrm{C}\right)$, the nanotube samples were placed in a vacuum $\left(\sim 10^{-4}\right.$ torr $)$ for approximately 5 - 10 minutes to rid nanopores of any trapped air. Approximately $1 \mathrm{ml}$ of $1 \%$ amoxicillin (Sigma) in phosphate buffered solution (PBS) pH 7.4 (Invitrogen) was loaded onto each sample placed individually in separate wells of a 12-well plate (Nunc). To ensure dissolution of the amoxicillin prior to loading, a few microliters of $1 \mathrm{~N}$ $\mathrm{HCl}$ was added until the solution became clear. The samples were incubated overnight to allow sufficient time for the drug to fully penetrate into the nanotube structure. The drug-loaded nanotube samples were washed $3 \mathrm{X}$ with ice cold PBS (to restrict diffusion from the reservoir). Next, the samples were individually placed in new wells (Nunc, 12-wells) incubated in a humidified $95 \%$ air $/ 5 \%$ v/v $\mathrm{CO}_{2}$ incubator at $37^{\circ} \mathrm{C}$ in $1 \mathrm{ml}$ fresh simulated body fluid (PBS was used in this study). The solution was collected at hourly time points initially (hours $1-6$ ) and daily time points thereafter (up to day 35 or 5 weeks) and
$1 \mathrm{ml}$ fresh PBS was added after each collection. Drug concentration was determined by measuring the absorbance of the fluid using a UV-VIS spectrophotometer at $\lambda$ $=230 \mathrm{~nm}$ (Biomate_3, Thermo Electron, Madison, WI). The assay was calibrated by use of PBS blanks and a standard curve was determined up to $2 \mathrm{mg} / \mathrm{ml}$ amoxicillin. Three replicates per experimental sample for each time point were measured and the average values \pm standard error (SE) was graphed to obtain release profiles, release rate, accumulation, and release kinetics.

\section{Results and Discussion}

The vertically aligned, periodic AAO nanotube structure used as a drug carrier is illustrated in the scanning electron microscopy (SEM) images in Figure 1 (top row). In contrast to conventional AAO nanopore structures, AAO nanotube unit cells are separated from each other while being loosely connected to each other, which is an interesting feature. In our nanotube samples, the nanotube center pore size $(\sim 20 \mathrm{~nm})$ is practically the same as the size of the voids (spacing between adjacent nanotubes) surrounding the center pore. This makes our AAO nanotube structure favorable due to larger surface area in terms of loading drugs or catalyst chemicals into the AAO nanotubes. The equal size of the center pore and voids was achieved by the relatively low anodizing voltage $(35 \mathrm{~V})$ which ensures both relatively small center pore size and interpore distance compared to the higher voltage evolution under the constant current anodization technique conducted by Zhao et al. [24]. The anodic current evolution during our nanotube fabrication is given in Figure 2.

To our knowledge, this is the first study to utilize the AAO nanotube structure for applications in drug elution. The nanotube film shows a highly ordered and uniform nanotube morphology and long-range order with nanotube height reaching $\sim 38 \mu \mathrm{m}$, which is the tallest used thus far in nanopore/nanotube ceramic alumina and titania drug elution studies. Physical details of the AAO nanotubes are portrayed in the chart shown in Figure 1 (bottom row). One of the advantages of our nanotube structure is the increased porosity $(\sim 25 \%)$ and the high surface-to-volume ratio. Here we show the surface area is increased by three orders of a magnitude by introducing the nanostructures on the surface, where a $1 \times 1 \mathrm{~cm}^{2}$ sample has perceivably $1830 \mathrm{~cm}^{2}$ of surface area. The interstitial space between the tube walls and the inner pore walls aid in this calculation and is an advantage over a traditional pore structure. Another benefit to our AAO drug release system is that we can design the nanotubes to match a desired pore size $(20-100 \mathrm{~nm})$, structural shape (pores vs. tubes), available porosity, and surface area which can help tailor, for instance by chemical 

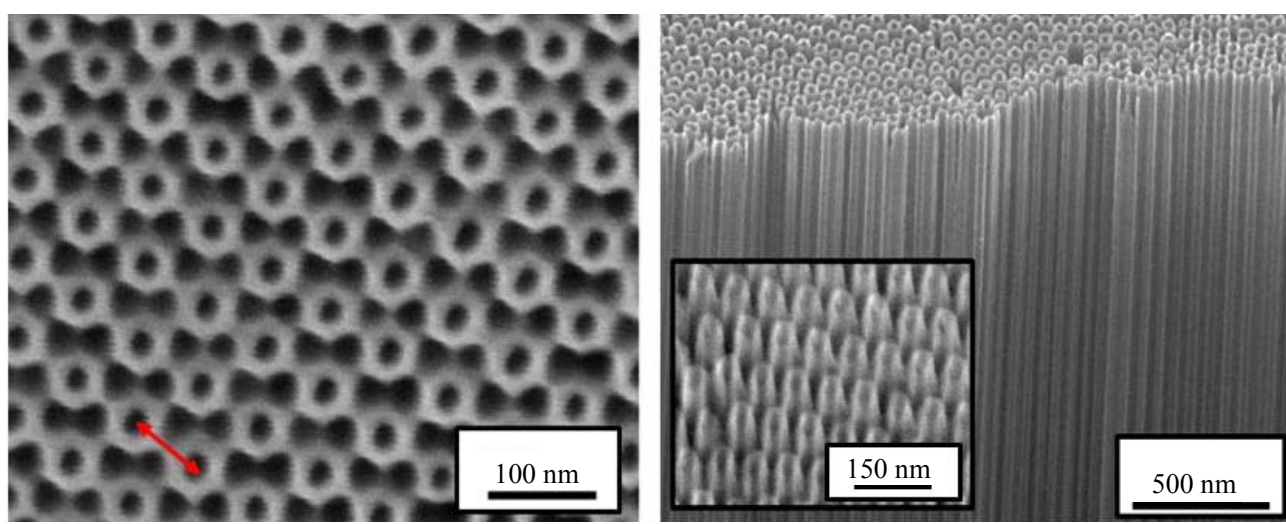

\begin{tabular}{|c|c|c|c|c|c|}
\hline $\begin{array}{c}\text { Nanotube } \\
\text { Height }(\mu \mathrm{m})\end{array}$ & Pore Size $(\mathrm{nm})$ & $\begin{array}{c}\text { Wall } \\
\text { Thickness } \\
(\mathrm{nm})\end{array}$ & $\begin{array}{c}\text { Nanotube } \\
\text { Center-to-Center } \\
\text { Distance }(\mathrm{nm})\end{array}$ & Porosity $(\%)$ & $\begin{array}{c}\text { Surface Area } \\
\left(\mathrm{cm}^{2}\right)\end{array}$ \\
\hline 38 & 20 & 15 & 73 & 25 & 1830 \\
\hline
\end{tabular}

Figure 1. Upper left: Top-view SEM image of vertically aligned periodic AAO nanotubes. Upper right: Oblique view (inset = higher magnification image). The table describes the physical dimensions of the nanotubes.

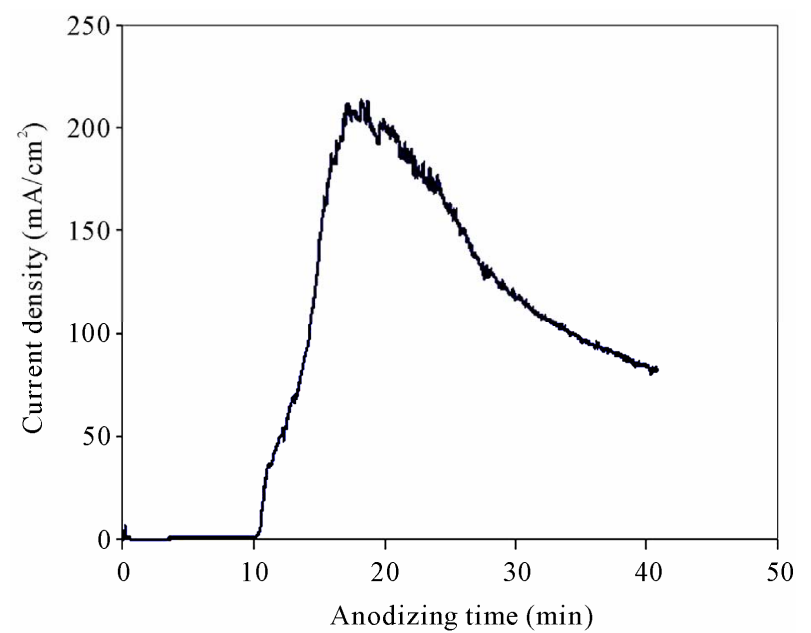

Figure 2. Representative anodic current evolution for AAO nanotube fabrication. Applied voltage was slowly increased from $25 \mathrm{~V}$ to $35 \mathrm{~V}$ and remained at $35 \mathrm{~V}$. Anodization was conducted for 30 minutes overall.

wet etching or pore widening techniques to specific implant needs and controlled release.

Factors such as adsorption properties (interactions between drug and matrix), pore size, pore connectivity, and pore geometry are just a few of the aspects to take into account when designing a controlled drug delivery system. It has been suggested that during AAO fabrication, stress cracking and other residual defects due to the oxidation volume expansion ( $\mathrm{Al}$ becoming $\mathrm{Al}_{2} \mathrm{O}_{3}$ ) may be present and these imperfections can leave charges on the surface, such as $\mathrm{Al}^{3+}$ and $\mathrm{O}^{2-}$ [25]. For the purposes of drug loading, this may aid in electrostatic adsorption of the drug molecules and help concentrate the drug within the nanotube "depots" so to speak.

For this study, amoxicillin (AMX), a common pharmaceutical antibiotic, is used as a model drug in the following AAO drug elution studies. The size of the AMX molecule is $\sim 0.8 \mathrm{~nm}$ [26], a reasonable size to enter and fill the $20 \mathrm{~nm}$ diameter pores and interstitial spaces in between the nanotubes. Preoperative oral administration of AMX has been proven to reduce the risk of implant failure [27,28], and local delivery of AMX during orthopedic surgery reduced the infections associated with open fractures [29], compound limb fractures [30], and with osteoinductive and osteoconductive bone-graft substitutes [29]. As well, local delivery of antibiotics was effective in reducing vascular infections from staphylococcal strains [31]. It can therefore be hypothesized that localized AMX elution from both orthopedic and vascular implants would be highly advantageous. With the more advanced drug delivery, controlled release system such as AAO nanotubes on implants, would help make improvements in delivery efficiency and localization which may also provide a solution for reducing dosages and help minimize toxic side effects and drug waste. The nanotube shape also has its advantages over a porous structure because it provides an optimal surface shape to allow for cellular adhesion sites for better cell attachment and proliferation [32], aiding in surface integration and cellular locking [33].

In terms of controlled release, it was found that the AAO nanotubes were cable of carrying cargo molecules (AMX, a small drug molecule) and releasing them in a physiological environment of the simulated body fluid, 
phosphate buffered solution (PBS). There are several types of controlled release devices and the AAO nanotube system presented here can be considered a drug diffusion-controlled release, where the entrapped drug diffuses out of a matrix at a defined rate [34]. An antibiotic release profile from the AAO nanostructures filled with AMX was obtained for over 5 weeks (35 days), illustrated in Figure 3. For a control experiment, electropolished aluminum, without a nanostructured surface, showed almost zero antibiotic release as expected (data not shown). This indicated that it was the nanotube design on the surface which created a reservoir for the AMX that was responsible for the drug release. Figure 3 shows the total amount of amoxicillin released as a function of time. A near steady release profile is achieved after the first week of release (after Day 7). The ideal release profile for most drugs would follow this type of a steady release rate so that the drug levels in the body remain constant while the drug is being administered [35]. The drug elution from the AAO nanotubes accomplishes the primary objective of a controlled release device which is to provide a sustained release for long periods of time on the order of days, weeks, even months.

In the inset graph of Figure 3, which shows the initial release of drug from the nanotubes in the first 6 hours of release, the highest "burst effect" is in the first hour with $\sim 13 \mu \mathrm{g}$ of drug release. The "burst effect" is often seen as controversy as to whether this is due to near-surface entrapped drug or surface-absorbed drug [36]. The initial burst and release of drug from the nanotubes may be related to several factors including 1) high relative top sur- face area 2) increased drug diffusivity through tube walls/channels and 3) high porosity. In addition, the specifics of the pore dimensions and their uniformity as well as subtle difference in physical form of the nanotubes may play a role at release during the initial short term release (first 7 days) before the steady elution (beyond 7 days in Figure 3). At this stage in release, the drugs are being released from the top portion of the film where the so called "matrix surface" becomes a factor. After seven days, however, it is suggested that the drugs are traveling from a distance that is farther down in the matrix and less likely to be affected by the very top surface. We have also studied drug release from AAO $\sim 20 \mathrm{~nm}$ and $\sim 40 \mathrm{~nm}$ pore (not tube) structures with the same film thickness or height as the nanotubes studied in this report (data not shown), however it has been suggested that it is the height of the pore, not the pore size, that changes the diffusion characteristics [15]. Varying film thickness will impart some of our future studies, but this report focuses on the unique geometry and beneficial properties of the AAO nanotube structure.

To further characterize the AMX release from the AAO nanotubes, Figures 4 and 5 illustrate the accumulative release (showing daily and weekly accumulation) and release rate per day over the 5 week elution study, respectively. A near steady release rate occurred over the course of the 5 weeks. This type of release would help maintain a drug level in a therapeutic window, avoiding the extremes of systematic drug over-dosages or under-dosages, eliminating the risks of adverse effects, drug waste, or being sub-therapeutic.

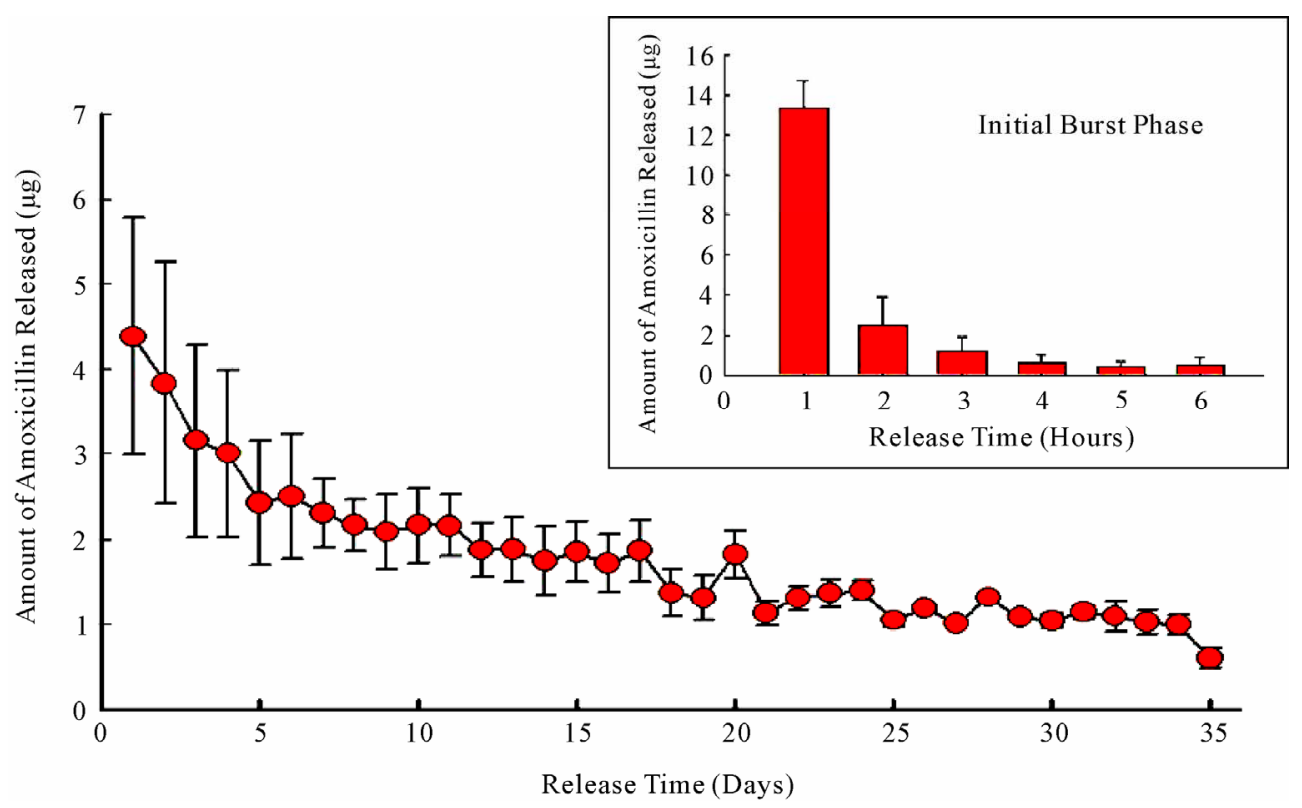

Figure 3. Absolute release rates of amoxicillin as a function of sampling time for the AAO nanotube drug carriers. The initial burst of drug from the surface is shown in the inset. The graphs show the mean $\pm \operatorname{SE}(n=3)$. 


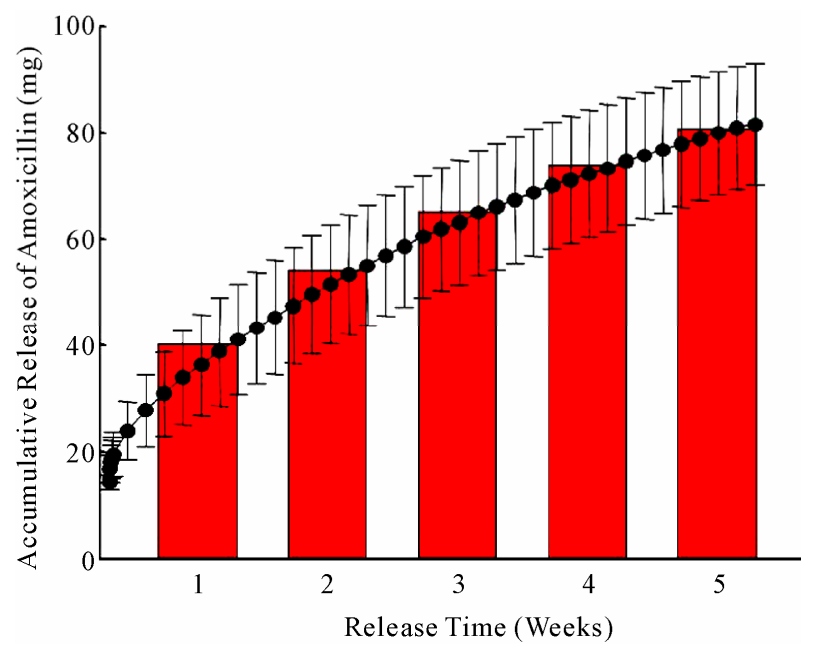

Figure 4. Accumulative amoxicillin released as a function of time. The graph shows mean $\pm \operatorname{SE}(n=3)$. The dotted line reveals the daily accumulation over time and the bars represent the average accumulation per week.

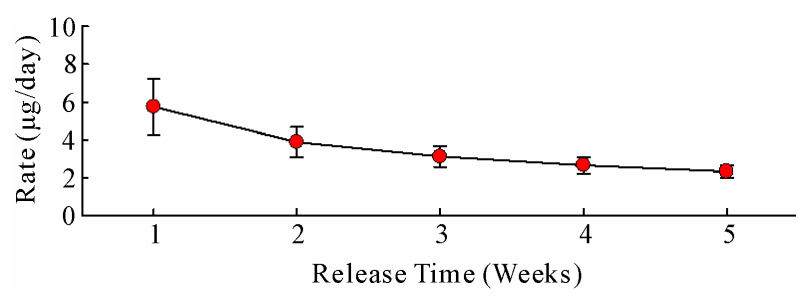

Figure 5. Daily release rate over time (normalized per weekly time point). The graph shows the mean \pm SE $(n=3)$.

When studying the drug release of molecules that have a size regime on the same scale as the matrix features, the basic principle of diffusion as a mixing process with solutes free to undergo Brownian motion in three dimensions may not necessarily apply, in at least on dimension, for the AAO nanotubes because the solute movement is physically constrained by the nanotube walls [37]. The AAO nanotube geometry may impose a rate limiting condition due to the length of the nanotube walls, because the length dictates how far the solute molecules have to travel to be released from the reservoir. While it is not possible to draw significant conclusions without varying the wall height of the AAO nanotubes, this in part will form some of our future work.

Many studies have observed that the release rate of a drug dispersed in a solid matrix (with no erosion of the matrix occurring) is proportional to the square root of time, as predicted by the Higuchi model [38-40]. It was determined that this was because the release rate is inversely proportional to the distance the drug must travel within the matrix to the matrix surface, since the diffusion distance increases with time, the release rate decreases with time [38]. The Higuchi equation follows,

$$
\frac{Q_{t}}{Q_{\infty}}=k \sqrt{t}
$$

where $\frac{Q_{t}}{Q_{\infty}}$ is the cumulative fractional release at time $t$ and $k$ is the release constant.

To identify the release rate mechanism and model the drug transport in the AAO nanotube system, the hypothesis was made that the release data obtained could be fitted using Equation (3) and the results are given in Figure 6, where the cumulative fractional AMX release, Equation (3), was plotted versus the square root of time. A near perfect linear fit was observed, demonstrating that the drug kinetics approximately follow the square root of time relationship. The chart in Figure 6 describes the linear fit. The mechanism of release is most likely attributable to a novel constrained diffusion mechanism provided by the AAO nanotube walls.

AAO films are simple to prepare and can be easily modified and structurally tailored. As well they are resistant to most physiologic and chemical reactions (bioinert), mechanically strong, and are considered biocompatible in vitro and in vivo. By utilizing AAO nanotubes as drug carriers, a variety of drugs can be loaded into the device reservoir in a range of physical states, including solutions and crystalline or micronized suspensions [37]. This flexibility with respect to encapsulated drugs provides options to substantially increase the load dose and duration of therapy, as well as stability of drugs that are unstable in certain biological fluids or different biochemical/acidic/alkaline environments. AAO films are

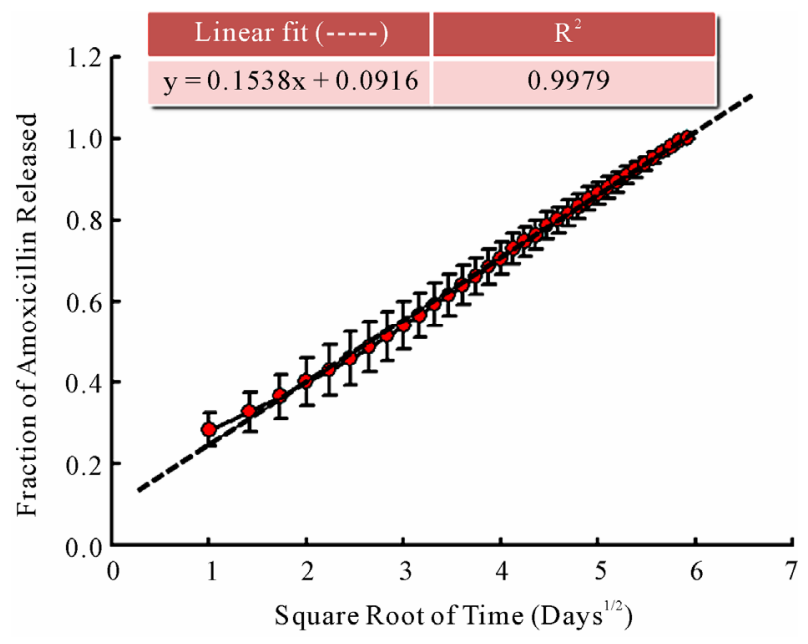

Figure 6. To assess the mechanism of drug release, a plot of fractional release $\left(\frac{Q_{t}}{Q_{\infty}}\right)$ vs. the square root of time was completed. A near perfect linear fit was observed and details are shown in the above table. 
structurally robust and will not swell or change its porosity under different $\mathrm{pHs}$ or temperatures [41]. Thus, AAO nanotube drug carriers can be used to address the problems associated with conventional drug therapies such as limited drug solubility, poor biodistribution, lack of selectivity and unfavorable pharmacokinetics [11]. Lastly, the potential for AAO nanotube arrays on implant surfaces will help mimic the complex geometries of natural tissue and will provide a porous template for the growth and maintenance of healthy cells and tissue [42], aiding in implant design as well as local delivery of therapeutics.

\section{Conclusions}

The controlled release of amoxicillin from anodic alumina oxide (AAO) nanotubes was investigated. The unique AAO nanotube morphology was fabricated using a simple two-step anodization process that resulted in highly uniform, structurally robust nanotubes. This is the first study utilizing the AAO nanotube geometry as a drug carrier and the diffusion characteristics including a drug release profile, drug accumulation plot, and release rate were acquired. The AAO nanotube carriers demonstrated controlled, sustained release of common antibiotic, amoxicillin for approximately 5 weeks. This study illustrates the potential advantages of using AAO nanotubes as a unique alternative in terms of therapeutics concepts for implant surface coatings.

\section{Acknowledgements}

This research was supported by Iwama endowed fund at UC San Diego, UC Discovery Grant No. ele08-128656/ Jin, and National Research Foundation (NRF) grant through World Class University Program (R33-2008000-10025-0).

\section{REFERENCES}

[1] H. Masuda and K. Fukuda, "Ordered Metal Nanohole Arrays Made by a 2-Step Replication of Honeycomb Structures of Anodic Alumina," Science, Vol. 268, No. 5216, 1995, pp. 1466-1468. doi:10.1126/science.268.5216.1466

[2] E. Gultepe, et al., "Nanoporous Inorganic Membranes or Coatings for Sustained Drug Delivery in Implantable Devices," Advanced Drug Delivery, Vol. 62, No. 3, 2010, pp. 305-315. doi:10.1016/j.addr.2009.11.003

[3] M. Darder, et al., "Encapsulation of Enzymes in Alumina Membranes of Controlled Pore Size," Thin Solid Films,. Vol. 495, No. 1-2, 2006, pp. 321-326. doi:10.1016/j.tsf.2005.08.285

[4] E. P. Briggs, et al., "Formation of Highly Adherent NanoPorous Alumina on Ti-Based Substrates: A Novel Bone Implant Coating," Journal of Materials Science-Materials in Medicine, Vol. 15, No. 9, 2004, pp. 1021-1029.

\section{doi:10.1023/B:JMSM.0000042688.33507.12}

[5] A. R. Walpole, et al., "Nano-Porous Alumina Coatings for Improved Bone Implant Interfaces," Materialwissenschaft Und Werkstofftechnik, Vol. 34, No. 12, 2003, pp. 1064-1068. doi:10.1002/mawe.200300707

[6] K. T. Huang, Z. Huang and D. B. Kim-Shapiro, "Nitric Oxide Red Blood Cell Membrane Permeability at High and Low Oxygen Tension," Nitric Oxide-Biology and Chemistry, Vol. 16, No. 2, 2007, pp. 209-216. doi:10.1016/j.niox.2006.11.002

[7] H. J. Kang, et al., "Controlled Drug Release Using Nanoporous Anodic Aluminum Oxide on Stent," Thin Solid Films, Vol. 515, No. 12, 2007, pp. 5184-5187. doi:10.1016/j.tsf.2006.10.029

[8] H. Wieneke, et al., "Synergistic Effects of a Novel Nanoporous Stent Coating and Tacrolimus on Intima Proliferation in Rabbits," Catheterization and Cardiovascular Interventions, Vol. 60, No. 3, 2003, pp. 399-407. doi:10.1002/ccd.10664

[9] D. W. Gong, et al., "Controlled Molecular Release Using Nanoporous Alumina Capsules," Biomedical Microdevices, Vol. 5, No. 1, 2003, pp. 75-80. doi:10.1023/A:1024471618380

[10] E. Gultepe, et al., "Sustained Drug Release from NonEroding Nanoporous Templates," Small, Vol. 6, No. 2, 2010, pp. 213-216. doi:10.1002/smll.200901736

[11] D. Losic and S. Simovic, "Self-ordered Nanopore And Nanotube Platforms for Drug Delivery Applications," Expert Opinion on Drug Delivery, Vol. 6, No. 12, 2009, pp. 1363-1381. doi:10.1517/17425240903300857

[12] C. Yao and T. J. Webster, "Prolonged Antibiotic Delivery from Anodized Nanotubular Titanium Using a Co-precipitation Drug Loading Method," Journal of Biomedical Materials Research Part B - Applied Biomaterials, Vol. 91B, No. 2, 2009, pp. 587-595. doi: $10.1002 / \mathrm{jbm} . b .31433$

[13] K. C. Popat, et al., "Influence of Engineered Titania Nanotubular Surfaces on Bone Cells," Biomaterials, Vol. 28, No. 21, 2007, pp. 3188-3197. doi:10.1016/j.biomaterials.2007.03.020

[14] K. C. Popat, et al., "Decreased Staphylococcus Epidermis Adhesion and Increased Osteoblast Functionality on Antibiotic-Loaded Titania Nanotubes," Biomaterials, Vol. 28, No. 32, 2007, pp. 4880-4888. doi:10.1016/j.biomaterials.2007.07.037

[15] L. Peng , et al., "Long-Term Small Molecule and Protein Elution from $\mathrm{TiO}_{2}$ Nanotubes," Nano Letters, Vol. 9, No. 5, 2009, pp. 1932-1936. doi:10.1021/n19001052

[16] S. Oh, et al., "Significantly Accelerated Osteoblast Cell Growth on Aligned $\mathrm{TiO}_{2}$ Nanotubes," Journal of Biomedical Materials Research Part A, Vol. 78, No. 1, 2006, pp. 97-103. doi:10.1002/jbm.a.30722

[17] S. Oh, et al., "Stem Cell Fate Dictated Solely by Altered Nanotube Dimension," Proceedings of the National Academy of Sciences of the United States of America, Vol. 106, No. 7, 2009, pp. 2130-2135. doi:10.1073/pnas.0813200106 
[18] K. S. Brammer, et al., "Improved Bone-Forming Functionality on Diameter-Controlled $\mathrm{TiO}_{(2)}$ Nanotube Surface," Acta Biomaterialia, Vol. 5, No. 8, 2009, pp. 3215 3223. doi:10.1016/j.actbio.2009.05.008

[19] L. M. Bjursten, L. Ramusson, S. Oh, et al., "Titanium Dioxide Nanotubes Enhance Bone Bonding in Vivo," Journal of Biomedical Materials Research Part A, Vol. 92A, No. 3, 2009, pp. 1218-1224.

[20] C. Ng, L. S. C. Chan, "Research Highlights: Highlights from the Latest Articles in Nanomedicine," Nanomedicine, Vol. 4, No. 4, 2009, pp. 381-383.

[21] W. Lee, et al., "Fast Fabrication of Long-Range Ordered Porous Alumina Membranes by Hard Anodization," Nature Materials, Vol. 5, No. 9, 2006, pp. 741-747. doi:10.1038/nmat1717

[22] W. Lee, et al., "Structural Engineering of Nanoporous Anodic Aluminium Oxide by Pulse Anodization of Aluminium," Nature Nanotechnology, Vol. 3, No. 4, 2008, pp. 234-239. doi:10.1038/nnano.2008.54

[23] K. Schwirn, et al., "Self-ordered Anodic Aluminum Oxide Formed by H2SO4 Hard Anodization," ACS Nano, Vol. 2, No. 2, 2008, pp. 302-310. doi:10.1021/nn7001322

[24] S. Y. Zhao, et al., "Novel Structure of AAO Film Fabricated by Constant Current Anodization," Advanced Materials, Vol. 19, No. 19, 2007, pp. 3004-3007. doi:10.1002/adma.200701284

[25] A. P. Li, et al., "Hexagonal Pore Arrays with a 50-420 nm Interpore Distance Formed by Self-Organization in Anodic Alumina," Journal of Applied Physics, Vol. 84, No. 11, 1998, pp. 6023-6026. doi:10.1063/1.368911

[26] M. Vallet-Regi, et al., "Hexagonal Ordered Mesoporous Material as a Matrix for the Controlled Release of Amoxicillin," Solid State Ionics, Vol. 172, No. 1-4, 2004, pp. 435-439. doi:10.1016/j.ssi.2004.04.036

[27] S. Geisler, "Orally Administered Amoxicillin Decreases the Risk of Implant Failures," The Journal of the American Dental Association, Vol. 140, No. 10, 2009, pp. 12941296.

[28] M. Esposito, et al., "Interventions for Replacing Missing Teeth: Antibiotics at Dental Implant Placement to Prevent Complications," Cochrane Database of Systematic Reviews, Vol. 16, No. 3, 2008, CD004152.

[29] A. A. Beardmore, et al., "Effectiveness of Local Antibiotic Delivery with an Osteoinductive and Osteoconductive Bone-Graft Substitute," Journal of Bone and Joint Surgery-American Volume, Vol. 87A, No. 1, 2005, pp. 107-112. doi:10.2106/JBJS.C.01670

[30] P. A. Ostermann, D. Seligson and S. L. Henry, "Local Antibiotic Therapy for Severe Open Fractures. A Review of 1085 Consecutive Cases," Journal of Bone and Joint Surgery (British Volume), Vol. 77, No. 1, 1995, pp. 9397.

[31] H. Turgut, et al., "Systemic and Local Antibiotic Prophy- laxis in the Prevention of Staphylococcus Epidermidis Graft Infection," Bmc Infectious Diseases, Vol. 5, 2005, p. 91. doi:10.1186/1471-2334-5-91

[32] K. Das, et al., "Surface Coatings for Improvement of Bone Cell Materials and Antimicrobial Activities of Ti Implants," Journal of Biomedical Materials Research Part B: Applied Biomaterials, Vol. 87, No. 2, 2008, pp. 455-460. doi:10.1002/jbm.b.31125

[33] S. Oh, et al., "Significantly Accelerated Osteoblast Cell Growth on Aligned $\mathrm{TiO}_{2}$ Nanotubes," Journal of Biomedical Materials Research A, Vol. 78, No. 1, 2006, pp. 97-103. doi:10.1002/jbm.a.30722

[34] W. Mark Saltzman and S. P. Baldwin, "Materials for Protein Delivery in Tissue Engineering," Advanced Drug Delivery Reviews, Vol. 33, No. 1-2, 1998, pp. 71-86. doi:10.1016/S0169-409X(98)00021-0

[35] J. M. Rosenholm and M. Linden, “Towards Establishing Structure-Activity Relationships for Mesoporous Silica in Drug Delivery Applications," Journal of Controlled Release, Vol. 128, No. 2, 2008, pp. 157-164. doi:10.1016/i.jconrel.2008.02.013

[36] G. Jiang, et al., "Assessment of Protein Release Kinetics, Stability and Protein Polymer Interaction of Lysozyme Encapsulated Poly(D,L-Lactide-Co-Glycolide) Microspheres," Journal of Controlled Release, Vol. 79, No. 1-3, 2002, pp. 137-145. doi:10.1016/S0168-3659(01)00533-8

[37] F. Martin, et al., "Tailoring width of Microfabricated Nanochannels to Solute Size Can be Used to Control Diffusion Kinetics," Journal of Controlled Release, Vol. 102, No. 1, 2005, pp. 123-133. doi:10.1016/j.jconrel.2004.09.024

[38] M. A. Bayomi, "Geometric Approach for Zero-Order Release of Drugs Dispersed in an Inert Matrix," Pharmaceutical Research, Vol. 11, No. 6, 1994, pp. 914-916. doi:10.1023/A:1018902513411

[39] T. Higuchi, "Rate of Release of Medicaments From Ointment Bases Containing Drugs in Suspension," Journal of Pharmaceutical Sciences, Vol. 50, No. 10, 1961, pp. 874-875. doi:10.1002/jps.2600501018

[40] T. Higuchi, "Mechanism of Sustained-Action Medication. Theoretical Analysis of Rate of Release of Solid Drugs Dispersed in Solid Matrices," Journal of Pharmaceutical Sciences, Vol. 52, No. 12, 1963, pp. 1145-1149. doi:10.1002/jps.2600521210

[41] I. Roy, et al., "Ceramic-Based Nanoparticles Entrapping Water-Insoluble Photosensitizing Anticancer Drugs: A Novel Drug-Carrier System for Photodynamic Therapy," Journal of the American Chemical Society, Vol. 125, No. 26, 2003, pp. 7860-7865. doi:10.1021/ja0343095

[42] K. C. Popat, et al., "Titania Nanotubes: A Novel Platform for Drug-Eluting Coatings for Medical Implants?" Small, Vol. 3, No. 11, 2007, pp. 1878-1881. doi: $10.1002 / \mathrm{smll} .200700412$ 